Revisão / Review

\title{
Análise morfológica, imunofenotípica e molecular na identificação da leucemia megacariocítica aguda (LMA-M7)
}

\author{
Morphologic, cytogenetic and molecular analyses in the identification of acute megakaryocytic \\ leukemia (AML-M7)
}

Mariela G. Farias ${ }^{1}$

Maristela B. Biermann²

\begin{abstract}
A leucemia megacariocítica aguda (LMA-M7) é um subtipo raro de leucemia mielóide aguda (LMA) que foi recentemente incorporada na classificação Franco-AmericanaBritânica (FAB). Ela representa 3\% a 5\% dos casos de LMA, sendo freqüentemente associada a mielofibrose e retrata um subtipo de mau prognóstico. O diagnóstico da LMA-M7 baseia-se, inicialmente, nas características morfológicas das células leucêmicas. O aspirado de medula óssea, ou biópsia, mostra uma população de células pleomórficas e basofílicas, podendo apresentar projeções citoplasmáticas (blebs). A utilização apenas de critérios morfológicos e citoquímicos não é suficiente para um diagnóstico correto, por isso faz-se necessária uma diferenciação de leucemia megacariocítica aguda com os outros subtipos de leucemia mielóide aguda, principalmente nos casos em que as células blásticas se apresentam indiferenciadas, como é o caso da leucemia mielóide aguda, minimamente diferenciada (LMA-M0), da leucemia mielóide aguda sem maturação (LMA-M1) e da leucemia linfóide aguda, subtipo L1 e L2. Sendo assim, a utilização de técnicas de imunofenotipagem é essencial para o diagnóstico diferencial, pois mostra uma população de células leucêmicas com ausência da maioria dos marcadores linfóides e mielóides de superfície, mas com expressão para os antígenos da linhagem megacariocítica: CD41a (complexo glicoproteíco IIb/ IIIa), CD42b (glicoproteína Ib) e/ou CD61 (glicoproteína IIIa), permitindo uma classificação correta em 98\% dos casos. Acrescenta-se também o estudo das anormalidades cromossômicas identificadas por técnicas de citogenética e análise molecular que passa a ser importante para a determinação do prognóstico e definição do regime terapêutico. Rev. bras. hematol. hemoter. 2007;29(4):387-393.
\end{abstract}

Palavras-chave: Leucemia megacariocítica aguda; morfologia; imunofenotipagem; análise molecular; diagnóstico.

\section{Introdução}

A leucemia megacariocítica aguda (LMA-M7) foi descrita pela primeira vez por Van Boros e Korenyi em $1931,{ }^{1}$ mas somente em 1985 foi definida como o sétimo subtipo da leucemia mielóide aguda, quando a classificação proposta pelo grupo cooperativo Franco-Americano-Britânico (FAB), baseada inicialmente em parâmetros morfológicos e cito- químicos, ${ }^{2}$ foi revisada e expandida, e passou a adotar critérios imunofenotípicos. ${ }^{3}$

A LMA-M7 é um subtipo raro - originada a partir de megacarioblastos primitivos - que corresponde a menos de 5\% dos casos de LMA, está freqüentemente associada a mielofibrose ${ }^{1,4-8}$ e exibe uma curva de incidência de distribuição com um pico bimodal, ou seja, um pico em crianças menores de três anos e outro em idosos. Ela é diagnosticada

${ }^{1}$ Unidade de Hematologia, Serviço de Patologia Clínica do Hospital de Clínicas de Porto Alegre.

${ }^{2}$ Laboratório Central, Irmandade da Santa Casa de Misericórdia de Porto Alegre.

Correspondência: Mariela Granero Farias

Unidade de Hematologia - Serviço de Patologia Clínica - Hospital de Clínicas de Porto Alegre

Rua Ramiro Barcelos, 2350 - Largo Eduardo Zaccaro Faraco

90035-903 - Porto Alegre-RS - Brasil

Tel.: (51) 2101-8674

E-mail:mgranero@uol.com.br 
pela demonstração de peroxidase plaquetária através da avaliação ultra-estrutural por microscopia eletrônica e/ou expressão imunológica de antígenos que são glicoproteínas (GP) específicas de membrana: GPIIb/IIIa (CD41a), GPIb (CD42b), GPIIIa (CD61), ${ }^{1,4-8}$ A LMA-M7 é a forma mais comum de leucemia mielóide aguda em crianças com Síndrome de Down (SD) e apresenta um ótimo prognóstico neste grupo de pacientes. Em outras crianças, a doença parece ser mais heterogênea e com pior fator prognóstico. 1,4,6,8,9

A LMA-M7 pode manifestar-se como uma leucemia de novo, leucemia secundária à quimioterapia, ou às síndromes mieloproliferativas e mielodisplásicas. ${ }^{5}$

O diagnóstico inicial das leucemias agudas consiste na diferenciação entre leucemia mielóide e linfóide, principalmente nos casos de leucemias indiferenciadas, pois as características morfológicas podem ser equívocas, requerendo estudos adicionais. ${ }^{10}$ Com base apenas em critérios morfológicos e citoquímicos, a LMA-M7 pode ser confundida com a leucemia linfóide aguda subtipo L2 (LLA-L2) ou, raramente, L1 e, até mesmo, com a leucemia mielóide aguda minimamente diferenciada (LMA-M0) e com a leucemia mielóide aguda sem maturação (LMA-M1).,7

A dificuldade de classificar alguns pacientes tem levado à busca de outros parâmetros, como a utilização de técnicas de imunofenotipagem. Esta tem importância diagnóstica e prognóstica em alguns subtipos FAB, sendo essencial para o diagnóstico de LMA-M7.4

A classificação imunofenotípica, a citogenética clássica e a análise molecular, associadas aos métodos tradicionais como a morfologia e a citoquímica, vieram contribuir para o diagnóstico e a classificação das leucemias agudas., 4,10

Este trabalho irá enfatizar a importância do diagnóstico laboratorial na LMA-M7, através da análise morfológica, citoquímica, imunofenotípica e análise molecular. Estas técnicas associadas a outros fatores clínicos e laboratoriais, permitem a identificação precisa deste subtipo de leucemia aguda, tendo importância fundamental na determinação do prognóstico e na escolha correta da abordagem terapêutica. ${ }^{10,11}$

\section{Diagnóstico laboratorial}

\section{Hemograma}

O sangue periférico pode apresentar pancitopenia com pronunciada leucopenia e anemia (normocítica e normocrômica), blastos leucêmicos circulantes são infreqüentes. ${ }^{12,13}$ A presença de fragmentos de megacariócitos pode ser indicativo de importante componente megacarioblástico na medula. ${ }^{8,11,13}$

\section{Medulograma}

O critério diagnóstico da LMA-M7 consiste na presença de $20 \%$, ou mais, de blastos do total de células nucleadas da medula óssea (TCN), excluindo linfócitos e plasmócitos, sendo mais de $50 \%$ dos blastos da linhagem megacariocítica. ${ }^{14}$ A informação clínica é essencial para uma melhor interpretação dos esfregaços de medula óssea. ${ }^{11}$

A medula óssea, freqüentemente, apresenta aumento das fibras de reticulina, devido à produção de citocinas e fatores de crescimento pelos megacarioblastos, ${ }^{15,16}$ o que pode dificultar a obtenção do aspirado medular; nesse caso, pode apresentar um número escasso de blastos. ${ }^{4,7,11,14}$ Outras vezes mostra-se hipercelular com infiltrado de blastos, formados por células, relativamente pequenas e monomórficas, ou por células grandes e pleomórficas. ${ }^{12}$

\section{Morfologia dos megacarioblastos}

Os blastos podem variar de pequenas células arredondadas com alta relação núcleo/citoplasma (N/C), assemelhando-se a linfoblastos de L1, a células um pouco maiores com relação N/C mais variada, assemelhando-se aos linfoblastos de L2. ${ }^{9,11,17}$ A cromatina, algumas vezes, é relativamente densa; outras vezes, o padrão aparenta ser bem mais delicado. ${ }^{11}$ O núcleo é geralmente arredondado ou com contorno discretamente irregular, com cromatina frouxa, contendo um a três nucléolos. ${ }^{14} \mathrm{O}$ citoplasma é tipicamente basofílico, e pode, ou não, ser vacuolado ou apresentar projeções citoplasmáticas (Figura 1). Mostra-se agranular, mas algumas vezes exibe uma fina granulação. .11,14,17 $^{2}$

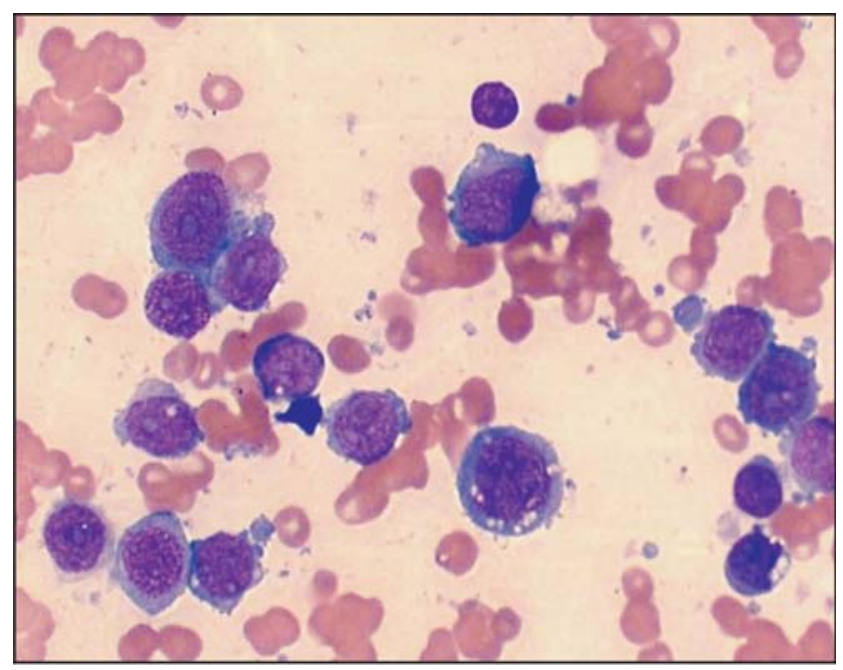

Figura 1. Distensão de medula óssea, corada com May-GrünwaldGiemsa (x500), exibe blastos com nucléolos evidentes, citoplasma basofílico, agranular, alguns com projeções citoplasmáticas e/ou vacúolos

\section{Citoquímica}

- Mieloperoxidase, Sudan black (SB) e cloroacetato esterase: os blastos M7 são negativos, exceto quando há associação com mieloblastos..$^{12}$ Essas colorações são insuficientes para diferenciar megacarioblastos de blastos linfóides. ${ }^{8,11}$ 
- Alfa-naftil acetato esterase (ANAE) e esterase inespecífica com inibição pelo fluoreto: megacarioblastos geralmente têm positividade distinta e bem localizada, em contraste com um padrão mais difuso das células monocíticas. ${ }^{11}$

- Ácido periódico de Schiff (PAS) e fosfatase ácida (FA): freqüentemente positivas e com padrão localizado. ${ }^{11}$ PAS é também positiva em eritroblastos e blastos linfóides. ${ }^{8}$

\section{Biópsia de medula óssea}

Em casos de aumentada fibrose medular, o diagnóstico deve ser baseado na biópsia de medula óssea, propiciando o aumento da visualização do número de blastos e, às vezes, de megacariócitos. Isso permite uma confirmação inequívoca de blastos da série plaquetária por meio do uso de marcadores imunológicos, uma vez que o aspirado, muitas vezes, é de difícil obtenção. ${ }^{11}$

\section{Imunofenotipagem}

As células hematopoéticas apresentam uma série de antígenos de superfície, intracitoplasmáticos e intranucleares; alguns estão associados a uma linhagem celular, outros indicam os diferentes estágios de maturação da célula. Pela imunofenotipagem por citometria de fluxo, os diferentes antígenos podem ser reconhecidos com o uso de anticorpos monoclonais específicos, que recebem uma designação de CD (clusters of differentiation), seguida de um número. ${ }^{18} \mathrm{~A}$ imunofenotipagem é considerada o método preferencial para a caracterização de algumas leucemias agudas, linfoblásticas ou não-linfoblásticas, particularmente nos casos de LMA do tipo M0, M1 e M7. ${ }^{18,19}$

Mais de 95\% das LMAs reagem com anticorpos monoclonais pan-mielóides, CD13, CD33 e CD117; estes são usados para diferenciar LMA de LLA. ${ }^{17}$ Eles possuem alta especificidade para células mielóides e são expressos em todos os subtipos morfológicos FAB da LMA. ${ }^{20} \mathrm{~A}$ LMAM7 pode não expressar CD13 ou CD33, ou expressar em baixa porcentagem dos blastos, com menos intensidade que na LMA clássica. Os megacarioblastos são positivos para os marcadores de células imaturas HLA-DR (80-90\% dos casos), CD34 e, em alguns casos, podem ser HLA-DR negativos ${ }^{4,8,11,14}$ e não expressam antígenos linfóides B. Em outros casos podem ser antígenos linfóides T (CD2 e/ou CD7) positivos. ${ }^{22}$

O diagnóstico da LMA-M7 requer positividade para os antígenos da linhagem megacariocítica CD41a (complexo glicoproteico IIb/IIIa), CD42b (glicoproteína Ib), CD61 (glicoproteína IIIa) ou antígenos relacionados ao fator VIII, , 4,6-9,20,22 confirmados por imunocitoquímica. ${ }^{7,11}$ Os antígenos CD41 e/ou CD61 são expressos já nas primeiras fases de megacarioblastos e o CD42 durante o processo de amadurecimento megacariocítico. ${ }^{7,11}$

Anormalidades cromossômicas

O estudo das alterações cromossômicas deve ser feito ao diagnóstico para prover-se a melhor classificação da LMA, a definição do prognóstico e a escolha terapêutica. Este estudo é útil também para eventual detecção posterior de doença residual mínima, de recaída ou de evolução clonal. As alterações cromossômicas são detectadas por técnicas de citogenética e por estudos moleculares. ${ }^{21}$

A porcentagem de cariótipo anormal, na M7 de novo, representa aproximadamente $80 \%$ dos casos em crianças e de $50 \%$ a $60 \%$ dos casos, em adultos. O grau de complexidade é maior em adultos do que em crianças, perfazendo três ou mais aberrações clonais. ${ }^{23}$

Nos adultos, não há anormalidade citogenética específica. ${ }^{22,24}$ Dentre os cariótipos complexos são encontrados 5/5q- e/ou $-7 / 7 q+$ em $70 \%$ a $80 \%$ dos casos; já alterações 3 q21 ou q26 são encontrados em $20 \%$ a $30 \%$ dos casos. Translocação $(9 ; 22)$ é outra alteração cromossômica recorrente na LMA-M7 de novo, ${ }^{22,24,25}$ e trissomias 19 e 21 podem ocorrer na leucemia de novo e na LMA-M7 secundária. ${ }^{22}$ Nas crianças, a t(1;22) (p13;q13) é encontrada em aproximadamente metade dos casos ${ }^{26,27}$ e ocorre em crianças com menos de 6 meses de idade. ${ }^{28,29}$ Os pacientes podem mostrar +21 sem necessidade de associação com $\mathrm{SD},+19$ e $+8 .{ }^{23,28,30,31} \mathrm{O}$ cariótipo pode ser normal em aproximadamente $10 \%$ dos casos. $^{23,31}$

Relatos de outras anormalidades, associadas pela primeira vez a LMA-M7, têm sido descritos na literatura, como a t(1;9)(p32;q34), ${ }^{32}$ tetrassomia $8,{ }^{33}$ trissomia $6,{ }^{34} 10 \mathrm{p} 12,{ }^{35}$ $\mathrm{t}(1 ; 21),{ }^{30}$ encontradas em crianças e a $\mathrm{t}(10 ; 22)$ em adultos. ${ }^{36}$ Down

Leucemia megacariocítica aguda na Síndrome de

Crianças com SD têm dez a vinte vezes maior risco de desenvolver leucemia aguda. ${ }^{37-40}$ A LMA-M7 é particularmente prevalente, com uma estimativa de risco 500 vezes maior, quando comparadas a crianças sem SD. ${ }^{16,41-44}$

A LMA-M7 associada à SD é um subgrupo homogêneo de leucemia, com apresentação clínica característica e excelente resposta à quimioterapia, baseada na trissomia do $21 .^{45,46} \mathrm{O}$ mecanismo pelo qual a trissomia do 21 predispõe o desenvolvimento de leucemia aguda é através da expressão aumentada de um gene ou genes no cromossomo 21, que estimula a proliferação anormal da stem cell hematopoética na infância. ${ }^{42,47}$

O gene GATA1 codifica um fator de transcrição hematopoético, que tem papel essencial para a diferenciação da linhagem eritróide e megacariocítica. ${ }^{47,48,49}$ Recentemente, mutações somáticas no éxon 2 desses genes codificadores do fator de transcrição hematopoético GATA1 foram encontradas nos blastos da leucemia megacariocítica, em pacientes com SD, indicando um papel significante dessas mutações no desenvolvimento da doença. ${ }^{37,44,47,49,50,51,52}$ Essas mutações são encontradas somente em indivíduos com SD, não sendo detectadas em outros tipos de leucemias agudas, ou em outros pacientes com leucemia megacariocítica que não apre- 
sentam SD. ${ }^{16,38,53}$ Há relato na literatura de somente um caso de mutação GATA1 em um adulto sem SD. ${ }^{54}$

Aproximadamente $10 \%$ das crianças com SD nascem com a chamada doença mieloproliferativa transitória (DMT), com cura espontânea em muitos casos, durante os três primeiros meses de vida, ${ }^{43,49,50,55,56}$ outras podem desenvolver formas fatais, com fibrose hepática e disfunção renal. ${ }^{48}$ Todavia, 30\% dos casos de DMT evoluem para leucemia megacariocítica aguda dentro dos quatro primeiros anos, sugerindo que alterações genéticas adicionais podem ocorrer durante a progressão da DMT para LMA-M7..$^{43,49} \mathrm{Na}$ DMT, os blastos são indistinguíveis pelas características morfológicas, citoquímicas e fenotípicas dos blastos da M7, e isso contribui para a hipótese de que a segunda doença é derivada da primeira. ${ }^{26,38,39,50,53,57,58}$

Recentes estudos demostram que mutações no gene GATA1, encontradas inicialmente em pacientes com LMAM7 com SD, também ocorrem na DMT, ${ }^{37,42,48,50,59,60}$ assim como mutações adquiridas no gene GATA1 têm sido relatadas. 37,60,61 $\mathrm{O}$ que os estudos ainda não esclareceram é porque o clone na DMT desaparece, subseqüentemente, em muitos pacientes, e em outros progridem para LMA-M7. ${ }^{59}$

\section{Discussão}

O diagnóstico diferencial da leucemia megacariocítica aguda, baseado apenas nas características morfológicas e citoquímicas, torna-se bastante difícil, principalmente entre as leucemias indiferenciadas LMA-M0, M1, e, destas, com a LLA L2; e em alguns casos, de L1. ${ }^{11}$ A dificuldade diagnóstica deve-se ao fato de, morfologicamente, os blastos da LMAM7 algumas vezes se apresentarem como células pequenas com cromatina frouxa e nucléolo evidente, citoplasma agranular, sem bastonetes de Auer, assemelhando-se aos blastos linfóides L1 e L2 da classificação $\mathrm{FAB}^{7}$ e, também, reagirem negativamente para colorações citoquímicas como mieloperoxidase e Sudan black. ${ }^{22}$

O achado de projeções citoplasmáticas, ao contrário do que se acreditava, não implica, necessariamente, em células da diferenciação megacariocítica. A secagem lenta de esfregaços de sangue periférico ou de medula óssea pode induzir à formação de grandes projeções citoplasmáticas. ${ }^{62}$

Segundo a classificação da Organização Mundial da Saúde (OMS), o limite de blastos, para a caracterização de uma leucemia aguda, passa a ser de $20 \%$, em confronto com o antigo 30\%, proposto pela classificação FAB. Para a OMS, pacientes com mais de $20 \%$ de blastos, na medula óssea, não diferem na sobrevida em relação a pacientes com mais de 30\% de blastos e têm um comportamento clínico similar. ${ }^{10,14,63,64}$ A OMS utiliza, como critério diagnóstico para a leucemia megacariocítica aguda, a presença de $50 \%$, ou mais, de blastos dessa linhagem; ao passo que a classificação FAB caracteriza esse subtipo pela presença de $30 \%$, ou mais, de megacarioblastos entre as células nucleadas da medula óssea. ${ }^{14}$

A imunofenotipagem identifica os antígenos celulares de superfície, citoplasmáticos e nucleares. Os mieloblastos, normalmente, não expressam os marcadores linfóides de membrana ou de citoplasma. A capacidade da citometria de fluxo em identificar a diferenciação mielóide aproxima-se de $98 \%$, mas, para tanto, deve-se usar um painel de anticorpos que assegure a distinção entre LMA e LLA, LMA minimamente diferenciada e leucemia megacariocítica, dentre outras. ${ }^{20,65}$ Assim, a imunofenotipagem com a identificação de glicoproteínas de membrana CD41, CD42 e/ou CD61 é considerada o método de escolha para o diagnóstico de leucemia megacariocítica aguda. ${ }^{5,6,7}$

Alguns casos de resultados falso-positivos, para os antígenos da série plaquetária CD41 e/ou CD61, podem ocorrer devido à aderência de plaquetas à superfície dos blastos. ${ }^{4,14,17,26,66}$ Deve-se ter o cuidado de coletar o material aspirado de medula óssea em frasco contendo EDTA, para minimizar a agregação de plaquetas. Amostras coletadas com outros anticoagulantes devem ser avaliadas por imunocitoquímica. ${ }^{11,13,26}$

A LMA-M7 é caracterizada por apresentar alta incidência de anormalidades cromossômicas e complexidade cariotípica em relação aos outros subtipos de leucemia mielóide aguda. Estas anormalidades mostram diferentes distribuições entre crianças e adultos. ${ }^{36}$ Rearranjos dos cromossomos 3 e 7 , assim como inv(3)(q21q26), t(3;3) (q21;q26) ${ }^{30}$ e monossomia do $7^{30,45}$ são descritos em pacientes com M7, porém estas anormalidades não são específicas, pois podem ocorrer em outros tipos de doenças hematopoéticas. A t(1;22)(p13;q13), comumente encontrada em bebês, a trissomia do 8, 19, e a trissomia do 21, alteração cromossômica numérica mais freqüente também estão associadas a LMA-M7. ${ }^{30}$

O fator de transcrição GATA1 codificado no cromossomo X é expresso em células de linhagem eritróide, megacariocítica, eosinofílica e mastócitos. ${ }^{53}$ É um regulador da diferenciação de células hematopoéticas e atua simultaneamente estimulando a diferenciação e suprimindo a proliferação celular. A perda do GATA1 acelera a expansão de células progenitoras de linhagem eritróide e megacariocítica. ${ }^{67} \mathrm{~A}$ ausência do GATA1 no megacariócito leva a um aumento na proliferação e deficiência na maturação dos progenitores megacariocíticos, bem como um reduzido número de plaquetas circulantes. As plaquetas produzidas não são totalmente funcionais e mostram morfologia anormal. ${ }^{68}$

O desenvolvimento de mutações no gene GATA1 parece estar envolvido na leucemogênese da SD e contribuir para a DMT e LMA-M7. ${ }^{38}$ Estas são dois estágios de uma única forma de leucemia que ocorre somente em células que são trissômicas para o cromossomo $21{ }^{43,48}$ Embora descrito por décadas, o mecanismo de remissão espontânea da DMT, e a progressão para leucemia megacariocítica aguda, ainda 
não estão bem esclarecidos e exigem futuros estudos. ${ }^{16,56}$ Aproximadamente, metade dos casos de pacientes com LMA-M7 realizam remissão completa com a quimioterapia convencional, mas poucos sobrevivem mais que três anos. ${ }^{1}$ A sobrevida média global da doença é estimada em quarenta semanas em adultos ${ }^{1,6} \mathrm{e}$ a sobrevida livre de doença é de dois anos em $14 \%$ das crianças. ${ }^{1,31}$ Enquanto crianças sem SD apresentam pior resposta ao tratamento, 70\% a 100\% dos casos de leucemia megacariocítica em crianças com SD podem ser curados pela quimioterapia. ${ }^{16}$ Os blastos dos SD são dez vezes mais sensíveis à citarabina e apresentam uma concentração intracelular significativamente maior que os blastos de crianças sem SD. ${ }^{54}$

A análise da leucemia em pacientes com SD tem destacado o papel do cromossomo 21na leucemogênese e no metabolismo das drogas quimioterápicas. O padrão de resposta clínica à terapia em pacientes com LMA-M7 é, provavelmente, multifatorial e pode estar ligado à detecção de mutações no GATA1 em pacientes com SD. ${ }^{69}$

O pior prognóstico não é completamente dependente de anormalidades citogenéticas, outros achados podem contribuir para um fator prognóstico desfavorável independente da alteração cromossômica encontrada. ${ }^{70}$

Atualmente, com a nova proposta da OMS, que utiliza dados citogenéticos/moleculares, associados a critérios clínicos, ${ }^{63,64} \mathrm{o}$ estudo de anormalidades cromossômicas é de grande importância para a classificação e para o diagnóstico das leucemias agudas. A técnica mais difundida é a análise citogenética, cujas informações podem ser complementadas e ampliadas com a técnica de hibridação in situ por fluorescência (FISH) e com o estudo molecular, através de reação em cadeia polimerase (PCR), ou PCR por transcriptase reversa (RT-PCR). ${ }^{14,21}$

Os métodos de biologia molecular vêm revolucionando o entendimento dos mecanismos envolvidos na etiopatogenia das leucemias. A compreensão de tais mecanismos proporciona uma rápida modificação nas abordagens diagnósticas e nos protocolos terapêuticos. ${ }^{71,72,73}$

\section{Abstract}

Acute megakaryocytic leukemia (AML-M7) is a rare subtype of acute myeloid leukemia (AML), which has recently been incorporated in the FAB (French-American-British) classification. It represents from 3 to $5 \%$ of $A M L$ cases, is frequently associated to myelofibrosis and is a subtype with poor prognosis. The diagnosis of AML-M7 is initially based on the morphologic characteristics of leukemic cells. Bone marrow aspirates or biopsy shows a population of pleomorphic and basophilic cells which may present cytoplasmatic blebs. Morphologic and cytochemical criteria are not enough for a correct diagnosis. It is necessary to differentiate acute megakaryocytic leukemia with other subtypes of acute myeloid leukemia mainly in cases in which blast cells are undifferentiated, as are the cases of AML-MO, AML-M1 and of acute lymphoid leukemia, subtypes L1 and L2. The use of immunopherotypic techniques is essential for a differential diagnosis showing a population of leukemia cells with the absence of most surface lymphoid and myeloid markers, yet relevant for the antigen of the megakaryocytic expression: CD41a (complex glycoproteic IIb/IIIa), CD42b (glycoprotein Ib) and/or CD61 (glycoprotein IIIa), there by providing the for correct classification in $98 \%$ of the cases. Chromosomal abnormalities identified by cytogenetic techniques and molecular analysis are important for determining the prognosis and definition of the therapheutic regime Rev. bras. hematol. hemoter. 2007;29(4):387-393.

Key words: Acute megakaryocytic leukemia;morphology; immunophenotype; molecular analysis; diagnosis.

\section{Referências Bibliográficas}

1. Garderet L, Labopin M, Gorin NC, Polge E, Baruchel A, Meloni G et al. Hematopoietic stem cell transplantation for de novo acute megakaryocytic leukemia in first complete remission: a retrospective study of the European Group for Blood and Marrow Transplantation (EBMT). Blood. 2005;105:405-9.

2. Bennett JM, Catovsky D, Daniel MT, Flandrin G, Galton DAG, Gralnick HR et al. Proposals for the classification of the acute leukaemias. Br J Haematol. 1976;33:451-8.

3. Bennet JM, Catovsky D, Daniel MT, Flandrin G, Galton DAG. Criteria for the diagnosis of acute leukemia of megakaryocytic lineage (M7). A report of the French-American-British cooperative assays. Ann Intern Med. 1985;103:460-2.

4. Martins SLR, Falcão RP. A importância da imunofenotipagem na leucemia mielóide aguda. Rev Assoc Med Bras. 2000; 46(1):57-62.

5. Tallman MS, Neuberg D, Bennett JM, Francois CJ, Paietta E, Wiernik PH et al. Acute megakaryocitic leukemia: the Eastern Cooperative Oncology Group Experience. Blood. 2000; 26(7): 2405-11.

6. Pagano L, Pulsoni A, Vignetti M, Mele L, Fianchi L, Petti MC et al. Acute megakaryoblastic leukemia: experience of GIMEMA trials. Leukemia. 2002;16:1622-6.

7. Kalleem Z, Crawford E, Pathan MH, Jasper L, Covinsky C, Johnson LR et al. Flow cytometric analysis of acute leukemias - Diagnostic utility and critical analysis of data. Pathology and Laboratory Medicine. 2003;127(1):42-8.

8. Greer JP, Foerster J, Lukens JN, Rodgers GM, Paraskevas F, Glader B. Wintrobe's Clinical Hematology. Chapter 79, 11 th ed, Philadelphia, 2004

9. Moriarty AT. Acute and chronic leukemias. In: Rodak BF. Diagnostic Hematology. 1997;23(1):343-4.

10. McKenna RW. Multifaceted approach to the diagnosis and classification of acute leukemias. Clin Chem. 2000;46(8) (B); 1252-9.

11. Oliveira RAG, Neto AP. Anemias e Leucemias. Conceitos Básicos e Diagnósticos por Técnicas Laboratoriais. São Paulo: Roca, 2004.

12. Bain BJ, Clark DM, Lampert IA. Acute myeloid leukemia, the myelodysplastic syndromes and histiocytic neoplasms. In: Bone Morrow-pathology. Blackwell Science. 1996;3(2):88-125.

13. Hutchison RE, Davey FR. Leucocytic disorders In: Henry JB et al. Clinical Diagnosis and Management by Laboratory Methods. Philadelphia: Suaunders Company, Cap 27, p. 4301-4304, 2001.

14. Rego EM, Falcão RP. Leucemia mielóide aguda - Diagnóstico: morfologia, imunofenótipo e citogenética. Ser Monogr Esc Bras Hematol. 2002;9:54-65.

15. Schmitz B, Wickenhauser C, Thiele J, Frimpong S, Brockbals C, Selbach B et al. Megakaryocyte induced fibroblast proliferation is 
enhanced by costimulation with IL-6/IL-3 and dependent on secretory and adhesion events. Leukemia Res. 1999;23:723-9.

16. Hitzler JK, Zipursky A. Origins of leukaemia in children with Down syndrome. Nat Rev Cancer. 2005;5:11-20.

17. Williams L, Wilkins. Clinical Laboratory Medicine. Philadelphia: Sarvier. 2 ed, p.1693, 2002.

18. Rocha MH. Leucemias. In Carrazza FR, Andriollo A. Diagnóstico Laboratorial em Pediatria. São Paulo: Sarvier, Cap 4, p.46-51. 2000.

19. Bahia DMM, Yamamoto M, Chauffaille MLLF, Kimura EYS, Bordin JO, Figueiras MAC et al. Aberrant phenotypes in acute myeloid leukemia: a high frequency and clinical significance. Haematologica. 2001;86(8):801-6.

20. Casasnovas RO, Slimane FK, Garand R, Faure GC, Campos L, Deneys $\mathrm{V}$ et al. Immunological classification of acute myeloblastic leukemias: relevance to patient outcome. Leukemia. 2003;17:515-27.

21. Braziel RM, Shipp MA, Feldman AL, Espina V, Winters M, Jaffe ES et al. Molecular diagnostics. Hematology. 2003;2003:279-93.

22. Cuneo A, Cavazzini F, Castoldi G. Acute megakaryoblastic leukemia (AmegL), M7 acute non lymphocytic leukemia (M7-ANLL). Atlas Genet Cytogenet Oncol Haematol. November 2003. URL: http:/ /www.infobiogen.fr/services/hromcancer/Anomalies/ M7ANLLID1100.html.

23. Dastugue N, Lafage-Pochitaloff M, Pagès MP, Radford I, Bastard C, Talmant $\mathrm{P}$ et al. Cytogenetic profile of childhood and adult megakarioblastic leukemia (M7): a study of the Groupe Français de Cytogénétique Hématologique (GFCH). Blood. 2002; 100(2): 618-26.

24. Berman E, Jhanwar S, McBride M, Strife A, Wisniewski D, Lambek $\mathrm{C}$ et al. Characterization of two novel sublines established from a human megakaryoblastic leukemia cell line transfected with p210 BCR-ABL. Leukemia Res. 2000;24:289-97.

25. Balatzenko G, Guenova M, Zechev S, Toshkov S. Acute megakaryoblastic leukemia with extreme thrombocytosis and p190 bcrabl rearrangement. Ann Hematol. 2004;83:381-5.

26. Hrusak O, Porwit-MacDonald A. Antigen expression patterns reflecting genotype of acute leukemias. Leukemia. 2002;16:1233-58.

27. Cen B, Selvaraj A, Bergess RC, Hitzler JK, Ma Z, Morris SW et al. Megakaryoblastic leukemia 1, a potent transcriptional coativator for serum response factor (SRF), is required for serum induction of SRF target genes. Mol Cell Biol. 2003;23(18):6597-608.

28. Trejo RMA, Aguilera RP, Nieto S, Kofman S. A t(1;22)(p13;q13) in four children with acute megakaryoblastic leukemia (M7), two with Down syndrome. Cancer Genet Cytogenet. 2000;120:160-2.

29. Mercher T, Coniat MB, Monni R, Mauchauffe M, Khac FN, Gressin $\mathrm{L}$ et al. Involvement of a human gene related to the Drosophila spen gene in the recurrent $t(1 ; 22)$ translocation of acute megakaryocytic leukemia. Proc Natl Acad Sci USA. 2001; 98(10):5776-9.

30. Paulien S, Coniat MBL, Berger R. Acute megakaryocytic leukemia with acquired polysomy 21 and translocation $t(1 ; 21)$. Ann Génét. 2000;43:99-104.

31. Athale UH, Razzouk BI, Raimondi SC, Tong X, Behm FG, Head DR et al. Biology and outcome of childhood acute megacaryoblastic leukemia: a single institution's experience. Blood. 2001;97(12): 3727-32.

32. Hasegawa D, Sano K, Kosaka Y, Hayakawa A, Kawagoe R, Kawasaki $\mathrm{K}$ et al. Acute megakaryoblastic leukemia in an infant with a novel $\mathrm{t}(1 ; 9)(\mathrm{p} 32 ; \mathrm{q} 34)$. Cancer Genet Cytogenet. 2000;122:59-62.

33. Aktas D, Tuncbilek E, Cetin M, Hicsonmez G. Tetrasomy 8 as a primary chromosomal abnormality in a child with acute megakaryoblastic leukemia: a case report and review of the literature. Cancer Genet Cytogenet. 2001;126:166-8.
34. McCullough SJ, Neat MJ, Power M, O’Marcaigh. Trisomy 6 in a child with acute megakaryoblastic leukemia (AML-M7). Cancer Genet Cytogenet. 2004;154:190-2.

35. Morerio C, Rapella A, Tassano E, Rosanda C, Panarello C. MLLMLLT10 fusion gene in pediatric acute megakaryoblastic leukemia. Leukemia Res. 2005;29:1223-6.

36. Kaloutsi V, Hadjileontis C, Tsatalas C, Sambani C, Kostopoulos I, Papadimitriou C. Occorrence of a variant Philadelphia translocation, $\mathrm{t}(10 ; 22)$, in de novo acute megakaryoblastic leukemia. Cancer Genet Cytogenet. 2004;152:52-5.

37. Wechsler J, Greene M, McDevitt MA, Anastasi J, Karp JE, Le Beau $\mathrm{MM}$ et al. Acquired mutation in GATA 1 in the megakaryoblastic leukemia of Down syndrome. Nat Genet. 2002;32:148-52.

38. Mundschau G, Gurbuxani S, Gamis AS, Greene ME, Arceci RJ, Crispino JD. Mutagenesis of GATA1 is an initiating event in Down syndrome leucemogenesis. Blood. 2003;101(11):4298-300.

39. Hirose Y, Kudo K, Kiyoi H, Hayashi Y, Naoe T, Kojima S. Comprehsive analysis of gene alterations in acute megakaryoblastic leukemia of Down's syndrome. Leukemia. 2003;17:2250-2.

40. Valladares A, Palma-Padilla V, Mejia-Aranguré JM, Guevara-Yánez R, Lerma-Reyes A, Salamanca-Gómez F. Cytogenetic studies in children with Down syndrome and acute leukemia. Leukemia Res. 2005;29:1241-6.

41. Stark B, Jeison M, Preudhomme C, Fenaux P, Ash S, Korek Y et al. Acquired trisomy 21 and distinct evolution in acute megakaryoblastic leukemia in young monozigotic twins. Br J Haematol. 2002;118:1082-6.

42. Xu G, Nagano M, Kanezaki R, Toki T, Hayashi Y, Taketani T et al. Frequent mutations in the GATA-1 gene in the transient myeloproliferative disorder of Down syndrome. Blood. 2003; 102(8):2960-8.

43. Zipursky A. Transient leukemia-a benign form of leukaemia in newborn infants. Br J Haematol. 2003;120:930-8.

44. Ahmed M, Stermberg A, Hall G, Thomas A, Smith O, O’Marcaigh A et al. Natural history of GATA 1 mutations in Down syndrome. Blood. 2004;103(7):2480-9.

45. Kobayashi K, Usami I, Kubota M, Nishio T, Kakazu N. Cromosome 7 abnormalities in acute megakaryoblastic leukemia associated with Down syndrome. Cancer Genet Cytogenet. 2005; 158:184-7.

46. Zeller B, Gustafsson G, Forestier E, Abrahamsson J, Clausen N, Heldrup J et al. Acute leukemia in children with Down syndrome: a population-based Nordic study. Br J Haematol. 2005;128:797-804.

47. Look AT. A leukemogenic twist for GATA 1. Nat Genet. 2002;32: $1-2$.

48. Greene ME, Mundschau G, Wechsler J, McDevitt M, Gamis A, Karp J et al. Mutations in GATA 1 in both transient myeloproliferative disorder and acute megakaryoblastic leukemia of Down syndrome. Blood Cells Mol Dis. 2003;31:351-6.

49. Li Z, Godinho FJ, Klusmann JH, Garriga-Canut M, Yu C, Orkin SH. Developmental stage-selective effect of somatically mutated leukemogenic transcription factor GATA 1. Nat Genet. 2005;37 (6):613-9.

50. Hitzler JK, Cheung J, Li Y, Scherer SW, Zipursky A. GATA1 mutations in transient leukemia and acute megakaryoblastic leukemia of Down syndrome. Blood. 2003;101(11):4301-4.

51. Gilliland DG, Jordan CT, Felix CA. The molecular basis of leukemia. Hematology 2004; 2004: 80-97.

52. Ge Y, Dombkowsky AA, LaFiura KM, Tatman D, Yedidi RS, Stout ML et al. Differential gene expression, GATA 1 target genes, and the chemotherapy sensitivity of Down syndrome megakaryocytic leukemia. Blood. 2006;107(4):1570-81. 
53. Gurbuxani S, Vyas P, Crispino JD. Recent insights into the mechanisms of myeloid leukemogenesis in Down syndrome. Blood. 2004;103(2):399-406.

54. Harigae H, Xu G, Sugawara T, Ishikawa I, Toki T, Ito E. The GATA 1 mutation in an adult patient with acute megakaryoblastic leukemia not accompanying Down syndrome. Blood. 2004; 103(8):3242-3.

55. Hasle H. Pattern of malignant disorders in individuals with Down's syndrome. Lancet Oncol. 2001;2:429-36.

56. Classen CF, Gnekow A, Debatin KM. Terminal differentation in vitro of patient-derived post-TMD megakaryoblastic AML cells. Ann Hematol. 2003;82:506-10.

57. Rainis L, Bercovich D, Strehi S, Teigier-Schiegel A, Stark B, Trka $\mathrm{J}$, et al. Mutations in exon 2 of GATA1 are early events in megakaryocytic malignancies associated with trisomy 21. Blood. 2003; 102(3):981-6.

58. Lighfoot J, Hitzler JK, Zipursky A, Albert M, Macgregor PF. Distinct gene signatures of transient and acute megakaryoblastic leukemia in Down syndrome. Leukemia. 2004;18:1617-23.

59. Kwong YL. Transient leukemia in newborn infants with trisomy 21. Br J Haematol. 2003;122:686-91.

60. Shimizu R, Kurhoa T, Ohneda O, Pan X, Ohneda K, Takahashi S et al. Leukemogenesis caused by incapacitated GATA-1 function. Mol Cell Biol. 2004;24(4):10814-25.

61. Groet J, McElwaine S, Spinelli M, Rinaldi A, Burtscher I, Mulligsn $\mathrm{C}$ et al. Acquired mutation in GATA 1 in neonates with Down's syndrome with transient myeloid disorder. Lancet 2003; 361: 1617-20.

62. Nguyen DT, Diamond LW, Braylan RC. Flow citometry in hematopathology. A visual approach to data analysis and interpretation. Humana Press. 2002;187-209.

63. Vardiman JW, Harris NL, Brunning RD. The World Health Organization (WHO) Classification of the myeloid neoplasms. Blood. 2002; 100(7):2292-302.

64. Harris NL, Jaffe ES, Diebold J, Flandrin G, Muller-Hermelink HK, Vardiman $\mathrm{J}$ et al. World Health Organization classification of neoplastic diseases of the hematopoietic and lymphoid tissues: Report of the Clinical Advisory Comittee meeting - Airlie House, Virginia, November 1997. J Clin Oncol. 1999; 17(12):3835-49.

65. Basso G, Buldini B, De Zen L, Orfao A. New methodologic approaches for immunophenotyping acute leukemias. Haematologica. 2001;87(7):675-92.

66. Betz SA, Foucar K, Head DR, Chen IM, Willman CL. False-positive flow cytometric platelet glycoprotein IIb/IIIa expression in myeloid leukemias secondary to platelet adherence to blasts. Blood. 1992; 79:2399-403

67. Takahashi S, Komeno T, Suwabe N, Yoh K, Nakajima O, Nishimura $S$ et al. Role of GATA-1 in proliferation and differentiation of definitive erythroid and megakaryocytic cells in vivo. Blood. 1998; 92(2):434-42.

68. Ferreira R, Ohneda K, Yamamoto M, Philipsen S. GATA 1 function, a paradigm for transcription factors in hematopoiesis. Mol Cell Biol. 2005;25(4):1215-27.

69. Ge Y, Stout ML, Tatman DA, Jensen TL, Buck SA, Thomas RL. GATA1, Cytidine deaminase, and the high cure rate of Down syndrome children with acute megakaryocytic leukemia. J Natl Cancer Inst. 2005;97(3):226-31.

70. Oki Y, Kantarijan MH, Zhou X, Cortes J, Faderl S, Verstovsek S et al. Adult acute megakaryocytic leukemia: an analysis of 37 patientes treated at M.D. Anderson Cancer Center. Blood. 2006; 107(3):880-4.

71. Mròzek K, Heinonen K, Bloomfield CD. Prognostic value of cytogenetic finding in adults with acute myeloid leukemia. Int J Hematol. 2000;72:261-71.

72. Haferlach T, Kern W, Schoch C, Schnittger S, Sauerland MC, Heinecke A. et al. A new prognostic score for patients with acute myeloid leukemia based on cytogenetics and early blast clearance in trials of German AML Cooperative Group. Haematologica. 2004; 89: 408-18.

73. Rizzatti EG, Zago MA. Aplicação da biologia molecular às leucemias agudas. Ser Monogr Esc Bras Hematol. 2002; 9: 1-14.

Avaliação: Editor e dois revisores externos Conflito de interesse: não declarado.

Recebido: 28/06/2006

Aceito após modificações: 28/03/2007 